Article

\title{
Hypervalent Iodine(III)-Induced Domino Oxidative Cyclization for the Synthesis of Cyclopenta[b]furans
}

\author{
Mei-Huey Lin *, Yu-Chun Chen, Shih-Hao Chiu, Yun-Fan Chen and Tsung-Hsun Chuang \\ Department of Chemistry, National Changhua University of Education, Changhua 50007, Taiwan; \\ stu01250008@gmail.com (Y.-C.C.); shihhao9239@yahoo.com.tw (S.-H.C.); evons44@gmail.com (Y.-F.C.); \\ th_chuang@hotmail.com (T.-H.C.) \\ * Correspondence: mhlin@cc.ncue.edu.tw; Tel.: +886-4-723-2105 (ext. 3543)
}

Academic Editors: Wesley Moran and Arantxa Rodríguez

Received: 17 November 2016; Accepted: 14 December 2016; Published: 21 December 2016

\begin{abstract}
A new strategy for cyclopenta[b]furan synthesis mediated by hypervalent iodine(III) has been described. The approach employs diacetoxyiodobenzene-induced initial dehydrogenation to a putative trienone intermediate and triggered sequential cycloisomerization to form the cyclo-penta[b]furan targets.
\end{abstract}

Keywords: cyclopenta[b]furan; hypervalent iodine(III); domino oxidative cyclization; diacetoxyiodobenzene; cycloisomerization

\section{Introduction}

Recent medicinal chemical studies have led to the identification of the cyclopenta[b]furan ring system as a potent inhibitor of CCR2, a chemokine receptor that plays an important role in inflammation-associated diseases $[1,2]$. Syntheses of the cyclopenta[b]furan core component have been achieved by employing palladium(0)-catalyzed intramolecular allylations (path a, Scheme 1), [3] and phosphine-catalyzed [3+2] cycloadditions of allenoates with $\beta, \gamma$-unsaturated $\alpha$-ketoesters (path $b$, Scheme 1) [4]. In addition, the construction of tricyclic cyclopenta[b]furan derivatives was elaborated through ethylenediammonium diacetate (EDDA)-catalyzed tandem Knoevenagel-polycyclization reactions between 1,3-dicarbonyl compounds and $\alpha, \beta, \gamma, \delta$-unsaturated aldehydes (path c, Scheme 1) or Lewis acid-promoted cycloisomerization of conjugated trienones (path $d$, Scheme 1) [5,6]. On the other hand, hypervalent hypervalent iodine species possess similar chemical properties and reactivity as heavy metal reagents but lower toxicity and an environmentally benign nature. Therefore, the utilization of hypervalent iodine in organic synthesis has been drawing intensive attention [7-12]. Among its reactions, the reactions of 1,3-dicarbonyl compounds with hypervalent iodine(III) species such as diacetoxyiodobenzene generate $\beta$-diketo iodonium ylides which behave as a 1,3-dipole, with an electrophilic center next to a carbonyl group. For example, the synthetic application of hypervalent iodine(III) in forming heterocycles involves the addition of $\beta$-diketo iodonium ylides to olefins to form fused dihydrofurans (path e, Scheme 1) $[13,14]$. Recent progress in the use of hypervalent iodine(III) reagents in organic synthesis has shown that diacetoxyiodobenzene-mediated dehydrogenation reactions of $\alpha$-alkyl- $\beta$-dicarbonyl compounds provide an alternative route to the Knoevenagel condensation $[15,16]$. Herein, we have devised a route for employing hypervalent iodine(III) in the synthesis of cyclopenta[b]furan derivatives $\mathbf{1}$ (Scheme 2). 
(a)

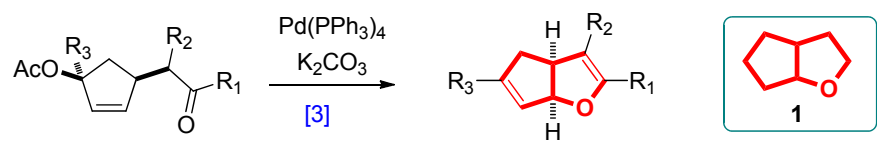

(b)

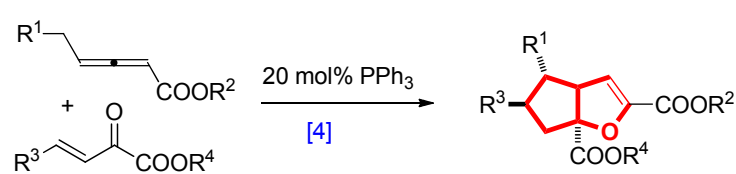

(c)

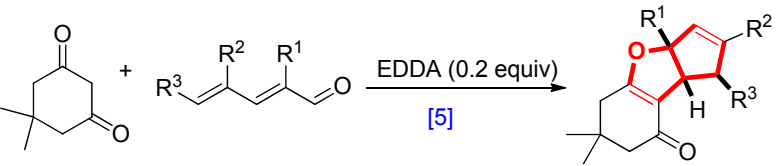

(d)

EDDA = ethylenediammonium diacetate

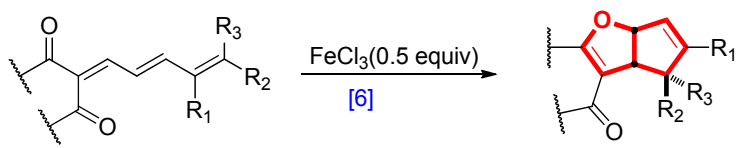

(e)

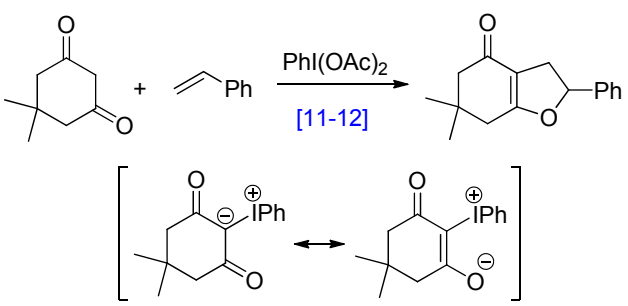

Scheme 1. Routes for the synthesis of cyclopenta[b]furans.

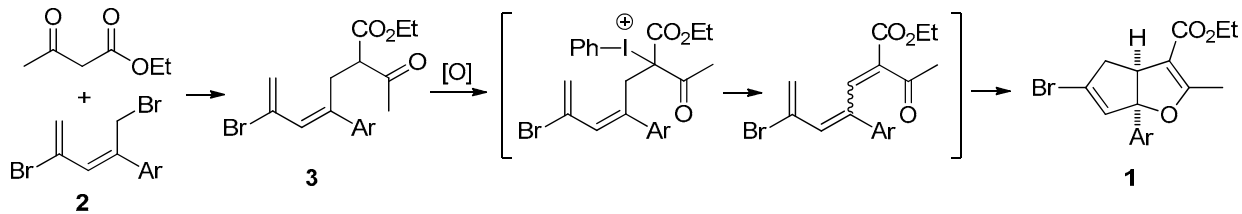

Scheme 2. Synthetic plans for the synthesis of cyclopenta[b]furan derivatives 1.

\section{Results and Discussions}

In the study described below, we have explored a method for the synthesis of cyclopenta[b]furans containing a stereogenic, quaternary, aryl-substituted bridgehead carbon. For the preparation of 2-aryl-1,4-dibromopenta-2,4-dienes 2 we used a literature procedure developed in our group [17]. The $\beta$-ketoesters linked with pentadiene tether 3 were prepared by $C$-alkylation of ethyl acetoacetate enolate with 2; however, the chromatographic purification of compounds 3 is difficult in most cases (Table 1). In fact, only $\mathbf{3 h}$ can be isolated in pure form by recrystallization after chromatographic purification. Crude analogues $\mathbf{3 a - g}$ as obtained after silica-gel plug treatment were carried directly into the next step, therefore, purity and exact yields of $\mathbf{3 a - g}$ are not given in Table 1 . In order to evaluate the feasibility of the new strategy for cyclopenta[b]furan synthesis, $\mathbf{3 b}$ serves as effective substrate in the reaction with diacetoxyiodobenzene that takes place by a one-pot, initial dehydrogenation to give a putative trienone intermediate and sequential cycloisomerization to form the cyclopenta[b]furan (Table 2). The results show that the putative trienone produced in these reactions is not isolable using chromatography, and at ambient temperature only the formation of the trienone was observed (Table 2, entries 1 and 8). At elevated temperature, the putative trienone underwent cycloisomerization 
to form cyclopenta[b]furans (entry 1 vs. entry 2, entry 7 vs. entry 8 ), reactions in which inorganic bases gave better yields than organic bases (entry 4 vs. entry 5, entry 8 vs. entry 9). In addition, the oxidation-cycloisomerization process happened smoothly when [bis(trifluoroacetoxy)iodo]benzene was employed. The ideal conditions for this process involve the use of diacetoxyiodobenzene and sodium carbonate in refluxing ethanol (Table 2, entry 7). Next, a variety of aryl-substituted substrates 3 were examined and Suzuki reactions were pursued in order to confirm the structural determination and the relative stereochemistry at the ring junction of the cyclopenta[b]furans $\mathbf{1}$ (Table 1 ).

Table 1. Synthesis of cyclopenta[b]furans.

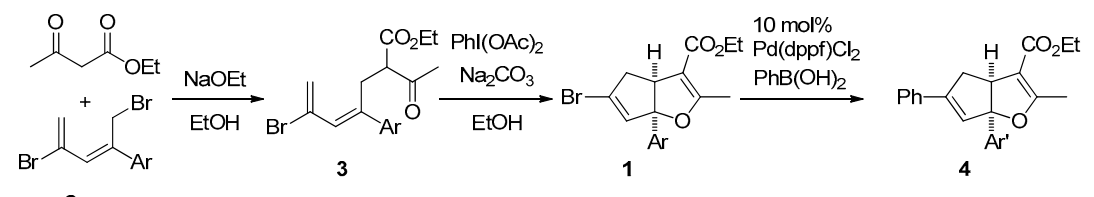

2

\begin{tabular}{|c|c|c|c|c|}
\hline Entry & Ar & 3, Yield (\%) & 1, Yield (\%) a & 4, Yield $(\%)^{c}$ \\
\hline 1 & & - & $\mathbf{1 a}, 72 ;(73)^{b}$ & $4 \mathbf{a}, \mathrm{Ar}^{\prime}=\mathrm{Ar}, 86$ \\
\hline 2 & & - & $\mathbf{1 b}, 78 ;(71)^{b}$ & $4 \mathrm{~b}, \mathrm{Ar}^{\prime}=4-\mathrm{PhC}_{6} \mathrm{H}_{4}, 85$ \\
\hline 3 & & - & $1 c, 90 ;(70)^{b}$ & $4 c, \mathrm{Ar}^{\prime}=\mathrm{Ar}, 86$ \\
\hline 4 & & - & $1 d, 70$ & $4 \mathrm{~d}, \mathrm{Ar}^{\prime}=\mathrm{Ar}, 84$ \\
\hline 5 & & - & $1 e, 65$ & $4 \mathbf{e}, \mathrm{Ar}^{\prime}=\mathrm{Ar}, 82$ \\
\hline 6 & & - & 1f, 63 & 4f, $\mathrm{Ar}^{\prime}=\mathrm{Ar}, 81$ \\
\hline 7 & & - & $1 \mathrm{~g}, 87$ & $4 \mathrm{~g}, \mathrm{Ar}^{\prime}=3-\mathrm{PhC}_{6} \mathrm{H}_{4}, 85$ \\
\hline 8 & & $3 g, 58$ & 1h, 64 & $4 h, \mathrm{Ar}^{\prime}=\mathrm{Ar}, 84$ \\
\hline
\end{tabular}

a Reagents and Conditions: $3(0.3 \mathrm{mmol}), \mathrm{PhI}(\mathrm{OAc})_{2}(0.6 \mathrm{mmol})$ and $\left.\mathrm{Na}_{2} \mathrm{CO}_{3}(0.66 \mathrm{mmol}) \mathrm{in} \mathrm{EtOH} \mathrm{(1.2} \mathrm{mL}\right)$. ${ }^{b} \mathrm{PhI}\left(\mathrm{OCOCF}_{3}\right)_{2}$ was employed instead of $\mathrm{PhI}(\mathrm{OAc})_{2}{ }^{\mathrm{c}} \mathbf{1}(0.5 \mathrm{mmol}), \mathrm{Cs}_{2} \mathrm{CO}_{3}(1.5 \mathrm{mmol}, 3.0 \mathrm{mmol}$ for $\mathbf{4 b}, \mathbf{4 g})$, $\mathrm{PhB}(\mathrm{OH})_{2}(0.75 \mathrm{mmol}, 1.5 \mathrm{mmol}$ for $\mathbf{4 b}, \mathbf{4 g}), \mathrm{Pd}(\mathrm{dppf}) \mathrm{Cl}_{2}(0.05 \mathrm{mmol})$ in dioxane/water $(5 / 1,0.5 \mathrm{~mL}) 100{ }^{\circ} \mathrm{C}$ for $2 \mathrm{~h}$.

Table 2. Reaction Optimizations ${ }^{\text {a }}$.

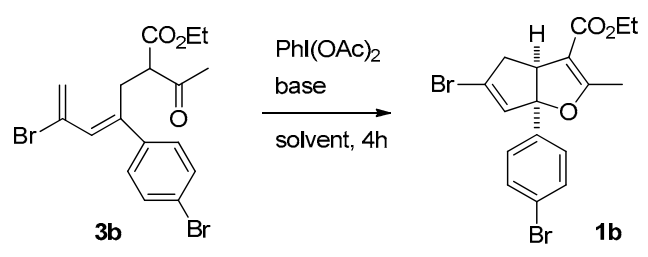

\begin{tabular}{cccc}
\hline Entry & Base & Solvent, Temp & Yield (\%) $^{\text {a }}$ \\
\hline 1 & $\mathrm{NaHCO}_{3}$ & $\mathrm{CH}_{3} \mathrm{CN}$, r.t. & 0 \\
2 & $\mathrm{NaHCO}_{3}$ & $\mathrm{CH}_{3} \mathrm{CN}$, reflux & 48 \\
3 & $\mathrm{Na}_{2} \mathrm{CO}_{3}$ & $\mathrm{CH}_{3} \mathrm{CN}$, reflux & 60 \\
4 & $\mathrm{~K}_{2} \mathrm{CO}_{3}$ & $\mathrm{CH}_{3} \mathrm{CN}$, reflux & 53 \\
5 & $\mathrm{DBU}_{2}$ & $\mathrm{CH}_{3} \mathrm{CN}$, reflux & 12 \\
6 & $\mathrm{Na}_{2} \mathrm{CO}_{3}$ & $\mathrm{PhCH}_{3}$, reflux & 26 \\
7 & $\mathrm{Na}_{2} \mathrm{CO}_{3}$ & EtOH, reflux & $78(71)^{\mathrm{b}}$ \\
8 & $\mathrm{Na}_{2} \mathrm{CO}_{3}$ & EtOH, r.t. & 0 \\
9 & $\mathrm{DMAP}$ & EtOH, reflux & 31 \\
\hline
\end{tabular}

a Reagents and Conditions: $3 \mathbf{b}(0.3 \mathrm{mmol}), \mathrm{PhI}(\mathrm{OAc})_{2}(0.6 \mathrm{mmol})$ and base $(0.66 \mathrm{mmol})$ in solvent $(1.2 \mathrm{~mL})$.

b $3 \mathbf{b}(0.3 \mathrm{mmol}), \mathrm{PhI}\left(\mathrm{OCOCF}_{3}\right)_{2}(0.6 \mathrm{mmol})$ and $\mathrm{Na}_{2} \mathrm{CO}_{3}(0.66 \mathrm{mmol})$ in $\mathrm{EtOH}(1.2 \mathrm{~mL})$ reflux. 
The stereochemistry of the fused-bicyclic ring system in $\mathbf{4 b}$ was shown to be cis using X-ray crystallographic analysis (Figure 1 and Supplementary Material) [18].

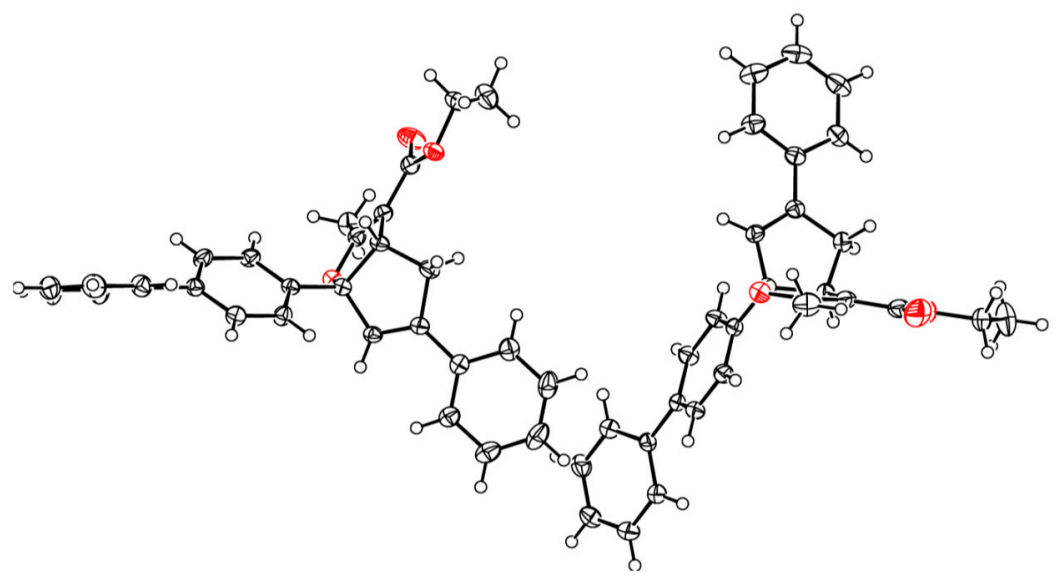

Figure 1. ORTEP plot of X-ray crystallographic data for $4 \mathbf{b}$.

A proposed plausible mechanism is shown in Scheme 3. First, the formation of putative trienone A through diacetoxyiodobenzene-mediated dehydrogenation reaction gave the newly formed $C-C$ double bond as $E / Z$-form mixtures or ones where the Z-isomer predominated [15]. However, only the $E$-isomer possesses the proper steric alignment to form the target cyclopenta[b]furans 1 . Therefore, a simple thermal cycloisomerization of the trienone to a cyclopenta[b]furan is not likely in this scenario. In the diacetoxyiodobenzene-mediated dehydrogenation process at elevated temperature, acetic acid produced during the reaction presumably plays an important role in promoting the sequential cycloisomerization from a cationic dienyl structure $\mathbf{B}$ to form the cyclopenta[b]furans.
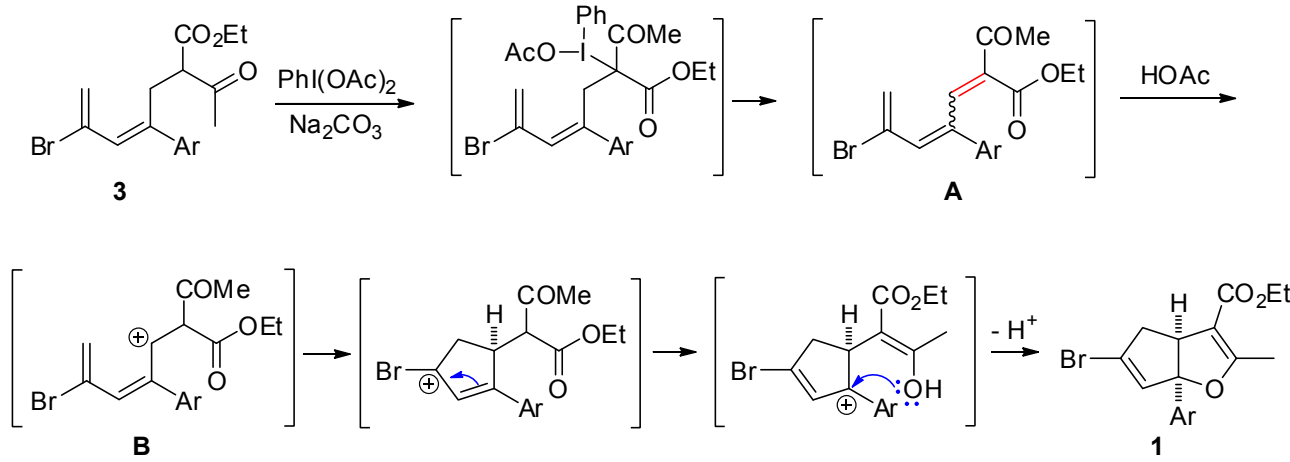

Scheme 3. Plausible Mechanism.

\section{Materials and Methods}

\subsection{General Information}

All commercially available chemicals were used without further purification. TLC analyses were run on a TLC glass plate (silica gel 60 F254, EMD Millipore, Darmstadt, Germany) and were visualized using UV and a solution of phosphomolybdic acid in ethanol (5 wt \%) or $p$-anisaldehyde stain. Flash chromatography was performed using silica gel (70-230 mesh, EMD Millipore). ${ }^{1} \mathrm{H}-\mathrm{NMR}$ spectra were recorded on a $300 \mathrm{MHz}$ spectrometer (Bruker AV-300, Bruker BioSpin GmbH, Karlsruhe, Germany). ${ }^{13} \mathrm{C}$-NMR spectra were recorded at $75 \mathrm{MHz}$ on the same instrument with complete proton decoupling (see Supplementary Material). Chemical shifts are reported relative to $\mathrm{CHCl}_{3}\left[\delta_{\mathrm{H}} 7.24, \delta_{\mathrm{C}}\right.$ (central line) 
77.0]. Mass spectra and high-resolution mass spectra were recorded on the Thermo/Finnigan Quest MAT Mass Spectrometer (Thermo Finnigan LLC, San Jose, CA, USA).

\subsection{Synthesis}

\subsubsection{General Procedure for the Synthesis of Cyclopenta[b]furans 1}

A mixture of bromodiene $2(0.3 \mathrm{mmol})$, ethyl acetoacetate $(0.3 \mathrm{mmol}, 39 \mathrm{mg})$, and NaOEt $(0.4 \mathrm{mmol}, 27 \mathrm{mg})$ in $\mathrm{EtOH}(2 \mathrm{~mL})$ was stirred at reflux overnight. The mixture was cooled to ambient temperature $\left(25-28{ }^{\circ} \mathrm{C}\right)$ and transferred to a separatory funnel, followed by addition of $\mathrm{Et}_{2} \mathrm{O}(5 \mathrm{~mL})$ and water $(10 \mathrm{~mL})$. The aqueous layer was back extracted with $\mathrm{Et}_{2} \mathrm{O}(2 \mathrm{~mL} \times 2)$. The combined organic layers were washed with brine $(5 \mathrm{~mL})$, dried over $\mathrm{MgSO}_{4}$, and concentrated in a rotary evaporator. The residue was purified by a silica gel plug using $\mathrm{Et}_{2} \mathrm{O} /$ hexanes $(10 / 1,10 \mathrm{~mL})$ as the eluent, followed by concentration to give the bromoester 3 . To a solution of bromoester $3(0.3 \mathrm{mmol}$, based on bromo-diene 2) in $\mathrm{EtOH}(1.2 \mathrm{~mL})$ was added $\mathrm{Na}_{2} \mathrm{CO}_{3}(0.6 \mathrm{mmol}, 64 \mathrm{mg})$ and $\mathrm{PhI}(\mathrm{OAc})_{2}(0.6 \mathrm{mmol}, 193 \mathrm{mg})$ at ambient temperature. The resulting mixture was heated to reflux under $\mathrm{N}_{2}$ for $3 \mathrm{~h}$. The reaction was then cooled room temperature and $\mathrm{H}_{2} \mathrm{O}(2 \mathrm{~mL})$ was added. $\mathrm{Et}_{2} \mathrm{O}(5 \mathrm{~mL})$ was added and the mixture was transferred to a separatory funnel. The aqueous layer was back extracted with $\mathrm{Et}_{2} \mathrm{O}(5 \mathrm{~mL} \times 2)$. The combined organic layers were washed with $2 \mathrm{~N} \mathrm{HCl}(2 \mathrm{~mL})$, dried over $\mathrm{Na}_{2} \mathrm{SO}_{4}$, filtered, and concentrated in a rotary evaporator. The residue was purified by silica gel chromatography using $\mathrm{Et}_{2} \mathrm{O} /$ hexanes $(1 / 10)$ as eluent to give the title products as yellow oils. Using this general procedure, the following title compounds were obtained:

Ethyl 5-bromo-2-methyl-6a-phenyl-4,6a-dihydro-3aH-cyclopenta[b]furan-3-carboxylate (1a). Yield $55 \mathrm{mg}$ (72\%). TLC $\left(\mathrm{Et}_{2} \mathrm{O} /\right.$ hexanes $\left.(1: 10)\right) R_{\mathrm{f}}=0.44 ;{ }^{1} \mathrm{H}-\mathrm{NMR}\left(\mathrm{CDCl}_{3}\right) \delta 1.25(\mathrm{t}, J=7.2 \mathrm{~Hz}, 3 \mathrm{H}), 2.28(\mathrm{~d}$, $J=1.5 \mathrm{~Hz}, 3 \mathrm{H}), 2.83(\mathrm{dt}, J=17.4,2.1 \mathrm{~Hz}, 1 \mathrm{H}), 3.15(\mathrm{ddd}, J=17.4,7.8,2.1 \mathrm{~Hz}, 1 \mathrm{H}), 3.74(\mathrm{dt}, J=7.8$, $1.5 \mathrm{~Hz}, 1 \mathrm{H}), 4.09-4.20(\mathrm{~m}, 2 \mathrm{H}), 5.98(\mathrm{t}, J=2.1 \mathrm{~Hz}, 1 \mathrm{H}), 7.25-7.35(\mathrm{~m}, 5 \mathrm{H}) ;{ }^{13} \mathrm{C}-\mathrm{NMR}\left(\mathrm{CDCl}_{3}\right) \delta 14.3$ $\left(\mathrm{CH}_{3}\right), 14.4\left(\mathrm{CH}_{3}\right), 46.9\left(\mathrm{CH}_{2}\right), 53.9(\mathrm{CH}), 59.6\left(\mathrm{CH}_{2}\right), 100.8(\mathrm{C}) 105.8(\mathrm{C}), 124.5(\mathrm{CH} \times 2), 128.0(\mathrm{CH})$, $128.6(\mathrm{CH} \times 2), 129.1(\mathrm{C}), 131.9(\mathrm{CH}), 142.0(\mathrm{C}), 165.5(\mathrm{C}), 167.0(\mathrm{C})$; IR (neat) 2977, 1698, $1644 \mathrm{~cm}^{-1}$; EI-MS $m / z$ (rel intensity) 350 ([M + 2] $\left.]^{+}, 10\right), 348\left([\mathrm{M}]^{+}, 10\right), 302$ (29), 223 (100); HRMS [M] $]^{+}$calcd. for $\mathrm{C}_{17} \mathrm{H}_{17} \mathrm{BrO}_{3}$ : 348.0361, found 348.0366.

Ethyl 5-bromo-6a-(4-bromophenyl)-2-methyl-4,6a-dihydro-3aH-cyclopenta[b]furan-3-carboxylate (1b). Yield $100 \mathrm{mg}(78 \%)$. TLC $\left(\mathrm{Et}_{2} \mathrm{O} /\right.$ hexanes $\left.(1: 10)\right) R_{\mathrm{f}}=0.43 ;{ }^{1} \mathrm{H}-\mathrm{NMR}\left(\mathrm{CDCl}_{3}\right) \delta 1.26(\mathrm{t}, J=7.2 \mathrm{~Hz}, 3 \mathrm{H}), 2.28$ $(\mathrm{d}, J=1.5 \mathrm{~Hz}, 3 \mathrm{H}), 2.80(\mathrm{dt}, J=17.4,1.8 \mathrm{~Hz}, 1 \mathrm{H}), 3.14(\mathrm{ddd}, J=17.4,7.8,1.8 \mathrm{~Hz}, 1 \mathrm{H}), 3.69(\mathrm{dt}, J=7.8$, $1.5 \mathrm{~Hz}, 1 \mathrm{H}), 4.06-4.28(\mathrm{~m}, 2 \mathrm{H}), 5.93(\mathrm{t}, J=1.5 \mathrm{~Hz}, 1 \mathrm{H}), 7.15(\mathrm{~d}, J=7.8 \mathrm{~Hz}, 2 \mathrm{H}), 7.46(\mathrm{~d}, J=7.8 \mathrm{~Hz}, 2 \mathrm{H})$; ${ }^{13} \mathrm{C}-\mathrm{NMR}\left(\mathrm{CDCl}_{3}\right) \delta 14.2\left(\mathrm{CH}_{3}\right), 14.4\left(\mathrm{CH}_{3}\right), 46.8\left(\mathrm{CH}_{2}\right), 53.9(\mathrm{CH}), 59.7\left(\mathrm{CH}_{2}\right), 100.3(\mathrm{C}) 105.9(\mathrm{C}), 122.0$ (C), $126.3(\mathrm{CH} \times 2), 129.6(\mathrm{C}), 131.5(\mathrm{CH}), 131.7(\mathrm{CH} \times 2), 141.1(\mathrm{C}), 165.4(\mathrm{C}), 166.8(\mathrm{C})$; IR (neat) 2976, 1703, $1488 \mathrm{~cm}^{-1}$; EI-MS $m / z$ (rel intensity) 428 ([M + 2] $\left.]^{+}, 19\right), 426\left([\mathrm{M}]^{+}, 10\right), 382$ (59), 301 (100); HRMS $[\mathrm{M}]^{+}$calcd. for $\mathrm{C}_{17} \mathrm{H}_{16} \mathrm{Br}_{2} \mathrm{O}_{3}$ : 425.9466, found 425.9462 .

Ethyl 5-bromo-6a-(4-chlorophenyl)-2-methyl-4,6a-dihydro-3aH-cyclopenta[b]furan-3-carboxylate (1c). Yield $104 \mathrm{mg}(90 \%)$. TLC $\left(\mathrm{Et}_{2} \mathrm{O} /\right.$ hexanes $\left.(1: 10)\right) R_{\mathrm{f}}=0.43 ;{ }^{1} \mathrm{H}-\mathrm{NMR}\left(\mathrm{CDCl}_{3}\right) \delta 1.25(\mathrm{t}, J=7.2 \mathrm{~Hz}, 3 \mathrm{H}), 2.28$ $(\mathrm{d}, J=1.5 \mathrm{~Hz}, 3 \mathrm{H}), 2.83(\mathrm{dt}, J=17.4,2.1 \mathrm{~Hz}, 1 \mathrm{H}), 3.13(\mathrm{ddd}, J=17.4,7.8,2.1 \mathrm{~Hz}, 1 \mathrm{H}), 3.68(\mathrm{dt}, J=7.8$, $1.5 \mathrm{~Hz}, 1 \mathrm{H}), 4.09-4.20(\mathrm{~m}, 2 \mathrm{H}), 5.93(\mathrm{t}, J=2.1 \mathrm{~Hz}, 1 \mathrm{H}), 7.20(\mathrm{~d}, J=8.7 \mathrm{~Hz}, 2 \mathrm{H}), 7.31(\mathrm{~d}, J=8.7 \mathrm{~Hz}, 2 \mathrm{H})$; ${ }^{13} \mathrm{C}-\mathrm{NMR}\left(\mathrm{CDCl}_{3}\right) \delta 14.2\left(\mathrm{CH}_{3}\right), 14.3\left(\mathrm{CH}_{3}\right), 46.8\left(\mathrm{CH}_{2}\right), 53.9(\mathrm{CH}), 59.6\left(\mathrm{CH}_{2}\right), 100.2(\mathrm{C}) 105.9(\mathrm{C}), 125.9$ $(\mathrm{CH} \times 2), 128.7(\mathrm{CH} \times 2), 129.5(\mathrm{C}), 131.5(\mathrm{CH}), 133.8(\mathrm{C}), 140.5(\mathrm{C}), 165.3(\mathrm{C}), 166.7(\mathrm{C})$; IR (neat) 2977, 1700, $1646 \mathrm{~cm}^{-1}$; EI-MS $\mathrm{m} / z$ (rel intensity) 384 ([M + 2] $\left.{ }^{+}, 15\right), 382$ ([M] $\left.]^{+}, 12\right), 338$ (44), 257 (100); HRMS $[\mathrm{M}]^{+}$calcd. for $\mathrm{C}_{17} \mathrm{H}_{16} \mathrm{BrClO}_{3}$ : 381.9971, found 381.9978 . 
Ethyl 5-bromo-2-methyl-6a-(p-tolyl)-4,6a-dihydro-3aH-cyclopenta[b]furan-3-carboxylate (1d). Yield $76 \mathrm{mg}$ (70\%). TLC $\left(\mathrm{Et}_{2} \mathrm{O} /\right.$ hexanes $\left.(1: 10)\right) R_{\mathrm{f}}=0.51 ;{ }^{1} \mathrm{H}-\mathrm{NMR}\left(\mathrm{CDCl}_{3}\right) \delta 1.25(\mathrm{t}, J=7.2 \mathrm{~Hz}, 3 \mathrm{H}), 2.28(\mathrm{~d}$, $J=1.5 \mathrm{~Hz}, 3 \mathrm{H}), 2.32(\mathrm{~s}, 3 \mathrm{H}), 2.82(\mathrm{dt}, J=17.4,1.8 \mathrm{~Hz}, 1 \mathrm{H}), 3.13(\mathrm{ddd}, J=17.4,7.8,1.8 \mathrm{~Hz}, 1 \mathrm{H}), 3.72(\mathrm{dt}$, $J=7.8,1.5 \mathrm{~Hz}, 1 \mathrm{H}), 4.09-4.20(\mathrm{~m}, 2 \mathrm{H}), 5.97(\mathrm{t}, J=1.8 \mathrm{~Hz}, 1 \mathrm{H}), 7.15(\mathrm{~s}, 4 \mathrm{H}) ;{ }^{13} \mathrm{C}-\mathrm{NMR}\left(\mathrm{CDCl}_{3}\right) \delta 14.3$ $\left(\mathrm{CH}_{3}\right), 14.4\left(\mathrm{CH}_{3}\right), 21.0\left(\mathrm{CH}_{3}\right), 46.8\left(\mathrm{CH}_{2}\right), 53.8(\mathrm{CH}), 59.5\left(\mathrm{CH}_{2}\right), 100.7(\mathrm{C}) 105.8(\mathrm{C}), 124.4(\mathrm{CH} \times 2)$, $128.9(\mathrm{C}), 129.2(\mathrm{CH} \times 2), 131.9(\mathrm{CH}), 137.8(\mathrm{C}), 139.0(\mathrm{C}), 165.6$ (C), $167.0(\mathrm{C})$; IR (neat) 2979, 1702, $1644 \mathrm{~cm}^{-1}$; EI-MS $m / z$ (rel intensity) $364\left([\mathrm{M}+2]^{+}, 5\right), 362\left([\mathrm{M}]^{+}, 6\right), 316(19), 237$ (100); HRMS [M] ${ }^{+}$ calcd. for $\mathrm{C}_{18} \mathrm{H}_{19} \mathrm{BrO}_{3}: 362.0518$, found 362.0515 .

Ethyl 5-bromo-6a-(4-methoxyphenyl)-2-methyl-4,6a-dihydro-3aH-cyclopenta[b]furan-3-carboxylate (1e). Yield $74 \mathrm{mg}(65 \%)$. TLC $\left(\mathrm{Et}_{2} \mathrm{O} /\right.$ hexanes $\left.(1: 10)\right) R_{\mathrm{f}}=0.48 ;{ }^{1} \mathrm{H}-\mathrm{NMR}\left(\mathrm{CDCl}_{3}\right) \delta 1.25(\mathrm{t}, J=7.2 \mathrm{~Hz}, 3 \mathrm{H}), 2.27(\mathrm{~d}$, $J=1.5 \mathrm{~Hz}, 3 \mathrm{H}), 2.81(\mathrm{dt}, J=17.4,1.8 \mathrm{~Hz}, 1 \mathrm{H}), 3.12(\mathrm{ddd}, J=17.4,7.8,1.8 \mathrm{~Hz}, 1 \mathrm{H}), 3.71(\mathrm{dt}, J=7.8,1.5 \mathrm{~Hz}$, $1 \mathrm{H}), 3.78(\mathrm{~s}, 3 \mathrm{H}), 4.06-4.22(\mathrm{~m}, 2 \mathrm{H}), 5.97(\mathrm{t}, J=1.8 \mathrm{~Hz}, 1 \mathrm{H}), 6.85(\mathrm{~d}, J=8.7 \mathrm{~Hz}, 2 \mathrm{H}), 7.18(\mathrm{~d}, J=8.7 \mathrm{~Hz}$, $2 \mathrm{H}) ;{ }^{13} \mathrm{C}-\mathrm{NMR}\left(\mathrm{CDCl}_{3}\right) \delta 14.3\left(\mathrm{CH}_{3}\right), 14.4\left(\mathrm{CH}_{3}\right), 46.8\left(\mathrm{CH}_{2}\right), 53.7(\mathrm{CH}), 55.3\left(\mathrm{CH}_{3}\right), 59.5\left(\mathrm{CH}_{2}\right), 100.6$ (C), $105.8(\mathrm{C}), 113.9(\mathrm{CH} \times 2), 125.8(\mathrm{CH} \times 2), 128.8(\mathrm{C}), 131.9(\mathrm{CH}), 134.1(\mathrm{C}), 159.3(\mathrm{C}), 165.6(\mathrm{C}), 166.9$ (C); IR (neat) 2981, 1698, $1643 \mathrm{~cm}^{-1}$; EI-MS $m / z$ (rel intensity) $380\left([\mathrm{M}+2]^{+}, 6\right), 378$ ([M] $\left.{ }^{+}, 6\right), 332$ (20), 253 (100); HRMS [M] $]^{+}$calcd. for $\mathrm{C}_{18} \mathrm{H}_{19} \mathrm{BrO}_{4}: 378.0467$, found 378.0458 .

Ethyl 5-bromo-6a-(3-methoxyphenyl)-2-methyl-4,6a-dihydro-3aH-cyclopenta[b]furan-3-carboxylate (1f). Yield $72 \mathrm{mg}(63 \%)$. TLC $\left(\mathrm{Et}_{2} \mathrm{O} /\right.$ hexanes $\left.(1: 10)\right) R_{\mathrm{f}}=0.45 ;{ }^{1} \mathrm{H}-\mathrm{NMR}\left(\mathrm{CDCl}_{3}\right) \delta 1.24(\mathrm{t}, J=7.2 \mathrm{~Hz}, 3 \mathrm{H}), 2.28$ $(\mathrm{d}, J=1.5 \mathrm{~Hz}, 3 \mathrm{H}), 2.82(\mathrm{dt}, J=17.4,1.8 \mathrm{~Hz}, 1 \mathrm{H}), 3.14(\mathrm{ddd}, J=17.4,7.8,1.8 \mathrm{~Hz}, 1 \mathrm{H}), 3.73(\mathrm{dt}, J=7.8$, $1.8 \mathrm{~Hz}, 1 \mathrm{H}), 3.79(\mathrm{~s}, 3 \mathrm{H}), 4.03-4.24(\mathrm{~m}, 2 \mathrm{H}), 5.95(\mathrm{t}, J=1.8 \mathrm{~Hz}, 1 \mathrm{H}), 6.79-6.86(\mathrm{~m}, 3 \mathrm{H}), 7.24-7.29(\mathrm{~m}, 1 \mathrm{H})$; ${ }^{13} \mathrm{C}-\mathrm{NMR}\left(\mathrm{CDCl}_{3}\right) \delta 14.2\left(\mathrm{CH}_{3}\right), 14.3\left(\mathrm{CH}_{3}\right), 46.8\left(\mathrm{CH}_{2}\right), 53.8(\mathrm{CH}), 55.2\left(\mathrm{CH}_{3}\right), 59.5\left(\mathrm{CH}_{2}\right), 100.6(\mathrm{C})$ $105.8(\mathrm{C}), 110.7(\mathrm{CH}), 112.7(\mathrm{CH}), 116.7(\mathrm{CH}), 129.0(\mathrm{C}), 129.7(\mathrm{CH}), 131.8(\mathrm{CH}), 143.6(\mathrm{C}), 159.7(\mathrm{C})$, 165.5 (C), 166.9 (C); IR (neat) 2981, 1702, $1644 \mathrm{~cm}^{-1}$; EI-MS $\mathrm{m} / \mathrm{z}$ (rel intensity) 380 ([M + 2] $\left.]^{+}, 10\right), 378$ $\left([\mathrm{M}]^{+}, 10\right), 332$ (21), 253 (100); HRMS [M] $]^{+}$calcd. for $\mathrm{C}_{18} \mathrm{H}_{19} \mathrm{BrO}_{4}$ : 378.0467, found 378.0461.

Ethyl 5-bromo-6a-(3-bromophenyl)-2-methyl-4,6a-dihydro-3aH-cyclopentalb]furan-3-carboxylate (1g). Yield $112 \mathrm{mg}(87 \%)$. TLC $\left(\mathrm{Et}_{2} \mathrm{O} /\right.$ hexanes $\left.(1: 10)\right) R_{\mathrm{f}}=0.53 ;{ }^{1} \mathrm{H}-\mathrm{NMR}\left(\mathrm{CDCl}_{3}\right) \delta 1.24(\mathrm{t}, J=7.2 \mathrm{~Hz}, 3 \mathrm{H}), 2.28(\mathrm{~d}$, $J=1.5 \mathrm{~Hz}, 3 \mathrm{H}), 2.83(\mathrm{dt}, J=17.7,2.1 \mathrm{~Hz}, 1 \mathrm{H}), 3.15(\mathrm{ddd}, J=17.7,7.8,2.1 \mathrm{~Hz}, 1 \mathrm{H}), 3.70(\mathrm{dt}, J=7.8,2.1 \mathrm{~Hz}$, $1 \mathrm{H}), 4.06-4.21(\mathrm{~m}, 2 \mathrm{H}), 5.95(\mathrm{t}, J=2.1 \mathrm{~Hz}, 1 \mathrm{H}), 7.15-7.20(\mathrm{~m}, 2 \mathrm{H}), 7.37-7.41(\mathrm{~m}, 2 \mathrm{H}) ;{ }^{13} \mathrm{C}-\mathrm{NMR}\left(\mathrm{CDCl}_{3}\right)$ 反 $14.2\left(\mathrm{CH}_{3}\right), 14.3\left(\mathrm{CH}_{3}\right), 46.7\left(\mathrm{CH}_{2}\right), 53.9(\mathrm{CH}), 59.6\left(\mathrm{CH}_{2}\right), 99.9(\mathrm{C}) 105.8(\mathrm{C}), 122.7(\mathrm{C}), 123.7(\mathrm{CH})$, 127.6 (CH), 129.6 (CH), $130.1(\mathrm{CH}), 131.0(\mathrm{CH}), 131.3(\mathrm{CH}), 144.2(\mathrm{C}), 165.2(\mathrm{C}), 166.6(\mathrm{C})$; IR (neat) 2979, $1704,1643 \mathrm{~cm}^{-1}$; EI-MS $m / z$ (rel intensity) 428 ([M + 2] $\left.]^{+}, 19\right), 426\left([\mathrm{M}]^{+}, 10\right), 382$ (59), 301 (100); HRMS $[\mathrm{M}]^{+}$calcd. for $\mathrm{C}_{17} \mathrm{H}_{16} \mathrm{Br}_{2} \mathrm{O}_{3}: 425.9466$, found 425.9460 .

Ethyl 5-bromo-2-methyl-6a-(naphthalen-2-yl)-4,6a-dihydro-3aH-cyclopenta[b]furan-3-carboxylate (1h). Yield $77 \mathrm{mg}(64 \%)$. TLC $\left(\mathrm{Et}_{2} \mathrm{O} /\right.$ hexanes $\left.(1: 10)\right) R_{\mathrm{f}}=0.48 ;{ }^{1} \mathrm{H}-\mathrm{NMR}\left(\mathrm{CDCl}_{3}\right) \delta 1.26(\mathrm{t}, J=7.2 \mathrm{~Hz}, 3 \mathrm{H}), 2.35$ $(\mathrm{d}, J=1.5 \mathrm{~Hz}, 3 \mathrm{H}), 2.90(\mathrm{dt}, J=17.4,1.8 \mathrm{~Hz}, 1 \mathrm{H}), 3.22(\mathrm{ddd}, J=17.4,7.8,1.8 \mathrm{~Hz}, 1 \mathrm{H}), 3.84(\mathrm{dt}, J=7.8$, $1.8 \mathrm{~Hz}, 1 \mathrm{H}), 4.11-4.23(\mathrm{~m}, 2 \mathrm{H}), 6.09(\mathrm{t}, J=1.8 \mathrm{~Hz}, 1 \mathrm{H}), 7.35(\mathrm{dd}, J=8.4,1.8 \mathrm{~Hz}, 1 \mathrm{H}), 7.44-7.51(\mathrm{~m}, 2 \mathrm{H})$, $7.74(\mathrm{~s}, 1 \mathrm{H}), 7.80-7.85(\mathrm{~m}, 3 \mathrm{H}) ;{ }^{13} \mathrm{C}-\mathrm{NMR}\left(\mathrm{CDCl}_{3}\right) \delta 14.3\left(\mathrm{CH}_{3}\right), 14.4\left(\mathrm{CH}_{3}\right), 46.9\left(\mathrm{CH}_{2}\right), 53.7(\mathrm{CH}), 59.6$ $\left(\mathrm{CH}_{2}\right), 100.9(\mathrm{C}) 105.9(\mathrm{C}), 112.6(\mathrm{CH}), 123.1(\mathrm{CH}), 126.2(\mathrm{CH}), 126.4(\mathrm{CH}), 127.6(\mathrm{CH}), 128.1(\mathrm{CH}), 128.7$ (CH), 129.3 (C), 131.8 (CH), 132.8 (C), 132.9 (C), 139.0 (C), 165.5 (C), 167.0 (C); IR (neat) 3058, 1700, 1637 $\mathrm{cm}^{-1}$; EI-MS $m / z$ (rel intensity) 400 ([M + 2] $\left.]^{+}, 11\right), 398$ ([M] $\left.]^{+}, 11\right), 273$ (99), 202 (100); HRMS [M]+ calcd. for $\mathrm{C}_{21} \mathrm{H}_{19} \mathrm{BrO}_{3}$ : 398.0518, found 398.0516.

\subsubsection{General Procedure for the Suzuki Reactions of Cyclopenta[b]furans 1}

To a solution of cyclopenta[ $b]$ furan $1(0.5 \mathrm{mmol})$ in dioxane $/ \mathrm{H}_{2} \mathrm{O}(5 \mathrm{~mL} / 1 \mathrm{~mL})$ was added $\mathrm{Cs}_{2} \mathrm{CO}_{3}$ (1.5 mmol, $489 \mathrm{mg}), \mathrm{PhB}(\mathrm{OH})_{2}(0.75 \mathrm{mmol}, 91 \mathrm{mg})$, and $\mathrm{Pd}(\mathrm{dppf}) \mathrm{Cl}_{2}(0.05 \mathrm{mmol}, 37 \mathrm{mg})$ at ambient temperature. The resulting mixture was vacuumed and purged with $\mathrm{N}_{2}$ for 3 times. The reaction was heated to $100{ }^{\circ} \mathrm{C}$ under $\mathrm{N}_{2}$ for $2 \mathrm{~h}$. The reaction was then cooled room temperature and concentrated in a rotary evaporator. EtOAc $(5 \mathrm{~mL})$ and $\mathrm{H}_{2} \mathrm{O}(1 \mathrm{~mL})$ were added and the mixture was transferred to 
a separatory funnel. The aqueous layer was back extracted with EtOAc $(5 \mathrm{~mL} \times 2)$. The combined organic layers were dried over $\mathrm{Na}_{2} \mathrm{SO}_{4}$, filtered, and concentrated in a rotary evaporator. The residue was purified by silica gel chromatography to give the title product. The following compounds were prepared using this method:

Ethyl 2-methyl-5,6a-diphenyl-4,6a-dihydro-3aH-cyclopenta[b]furan-3-carboxylate (4a). Yield $149 \mathrm{mg}(86 \%)$. A yellow oil; TLC (EtOAc/hexanes (1:10)) $R_{\mathrm{f}}=0.50 ;{ }^{1} \mathrm{H}-\mathrm{NMR}\left(\mathrm{CDCl}_{3}\right) \delta 1.29(\mathrm{t}, J=7.2 \mathrm{~Hz}, 3 \mathrm{H}), 2.31$ $(\mathrm{s}, 3 \mathrm{H}), 3.02(\mathrm{dt}, J=17.1,2.1 \mathrm{~Hz}, 1 \mathrm{H}), 3.29(\mathrm{ddd}, J=17.1,7.8,2.1 \mathrm{~Hz}, 1 \mathrm{H}), 3.88(\mathrm{dt}, J=7.8,1.5 \mathrm{~Hz}, 1 \mathrm{H})$, 4.11-4.26 (m, 2H), $6.23(\mathrm{t}, J=2.1 \mathrm{~Hz}, 1 \mathrm{H}), 7.26-7.39(\mathrm{~m}, 8 \mathrm{H}), 7.54(\mathrm{~d}, J=7.8 \mathrm{~Hz}, 2 \mathrm{H}) ;{ }^{13} \mathrm{C}-\mathrm{NMR}\left(\mathrm{CDCl}_{3}\right)$ $\delta 14.4,40.2,52.8,59.3,102.3,106.4,124.6,125.5,126.4,127.5,128.4,128.5,134.7,143.1,146.5,165.9,166.6$; IR (neat) 2977, 1698, $1644 \mathrm{~cm}^{-1}$; EI-MS m/z (rel intensity) 346 ([M] $\left.]^{+}, 12\right), 300$ (100), 257 (30), 229 (47); HRMS [M] $]^{+}$calcd. for $\mathrm{C}_{23} \mathrm{H}_{22} \mathrm{O}_{3}$ : 346.1569, found 346.1561.

Ethyl 6a-([1,1'-biphenyl]-4-yl)-2-methyl-5-phenyl-4,6a-dihydro-3aH-cyclopenta[b]furan-3-carboxylate (4b). Yield $180 \mathrm{mg}(85 \%)$. A colorless solid, m.p. $118-120{ }^{\circ} \mathrm{C}$; TLC (EtOAc/hexanes $\left.(1: 10)\right) R_{\mathrm{f}}=0.35 ;{ }^{1} \mathrm{H}-\mathrm{NMR}$ $\left(\mathrm{CDCl}_{3}\right) \delta 1.28(\mathrm{t}, J=7.2 \mathrm{~Hz}, 3 \mathrm{H}), 2.31(\mathrm{~s}, 3 \mathrm{H}), 3.02(\mathrm{dt}, J=17.1,1.8 \mathrm{~Hz}, 1 \mathrm{H}), 3.31(\mathrm{ddd}, J=17.1,6.0$, $2.1 \mathrm{~Hz}, 1 \mathrm{H}), 3.91(\mathrm{dt}, J=6.0,1.8 \mathrm{~Hz}, 1 \mathrm{H}), 4.10-4.25(\mathrm{~m}, 2 \mathrm{H}), 6.25(\mathrm{t}, J=1.8 \mathrm{~Hz}, 1 \mathrm{H}), 7.30-7.45(\mathrm{~m}, 8 \mathrm{H})$, 7.53-7.55 (m, 6H) ${ }^{13} \mathrm{C}-\mathrm{NMR}\left(\mathrm{CDCl}_{3}\right) \delta 14.3,40.1,52.8,59.3,102.2,106.4,125.0,125.4,126.3,126.9,127.1$, 127.2, 128.3, 128.4, 128.6, 134.6, 140.4, 140.5, 142.1, 146.5, 165.8, 166.5; IR (neat) 3031, 1700, $1637 \mathrm{~cm}^{-1}$; EI-MS m/z (rel intensity) $422\left([\mathrm{M}]^{+}, 10\right), 376$ (100), 333 (13), 305 (23); HRMS [M] $]^{+}$calcd. for $\mathrm{C}_{29} \mathrm{H}_{26} \mathrm{O}_{3}$ : 422.1882 , found 422.1878 .

Ethyl 6a-(4-chlorophenyl)-2-methyl-5-phenyl-4,6a-dihydro-3aH-cyclopenta[b]furan-3-carboxylate (4c). Yield $164 \mathrm{mg}(86 \%)$. A yellow oil; TLC (EtOAc/hexanes $(1: 10)) R_{\mathrm{f}}=0.43 ;{ }^{1} \mathrm{H}-\mathrm{NMR}\left(\mathrm{CDCl}_{3}\right) \delta 1.31(\mathrm{t}$, $J=7.2 \mathrm{~Hz}, 3 \mathrm{H}), 2.32(\mathrm{~s}, 3 \mathrm{H}), 3.04(\mathrm{dt}, J=17.1,2.1 \mathrm{~Hz}, 1 \mathrm{H}), 3.30(\mathrm{ddd}, J=17.1,7.8,2.1 \mathrm{~Hz}, 1 \mathrm{H}), 3.84(\mathrm{dt}$, $J=7.8,1.8 \mathrm{~Hz}, 1 \mathrm{H}), 4.13-4.29(\mathrm{~m}, 2 \mathrm{H}), 6.19(\mathrm{t}, J=1.8 \mathrm{~Hz}, 1 \mathrm{H}), 7.27-7.40(\mathrm{~m}, 7 \mathrm{H}), 7.54(\mathrm{~d}, J=7.8 \mathrm{~Hz}, 2 \mathrm{H})$; ${ }^{13} \mathrm{C}-\mathrm{NMR}\left(\mathrm{CDCl}_{3}\right) \delta 14.4,40.1,52.9,59.4,101.8,106.4,124.6,125.0,126.1,128.4,128.5,128.6,133.3,134.5$, 141.7, 146.9, 165.7, 166.4; IR (neat) 2979, 1698, $1643 \mathrm{~cm}^{-1}$; EI-MS $m / z$ (rel intensity) $382\left([\mathrm{M}+2]^{+}, 4\right)$, $380\left([\mathrm{M}]^{+}, 10\right), 334(100), 228(36)$; HRMS [M] ${ }^{+}$calcd. for $\mathrm{C}_{23} \mathrm{H}_{21} \mathrm{ClO}_{3}: 380.1179$, found 380.1184.

Ethyl 2-methyl-5-phenyl-6a-(p-tolyl)-4,6a-dihydro-3aH-cyclopenta[b]furan-3-carboxylate (4d). Yield $151 \mathrm{mg}$ (84\%). A yellow oil; TLC (EtOAc/hexanes (1:10)) $R_{\mathrm{f}}=0.60 ;{ }^{1} \mathrm{H}-\mathrm{NMR}\left(\mathrm{CDCl}_{3}\right) \delta 1.31(\mathrm{t}, J=7.2 \mathrm{~Hz}, 3 \mathrm{H})$, $2.32(\mathrm{~s}, 3 \mathrm{H}), 2.36(\mathrm{~s}, 3 \mathrm{H}), 3.03(\mathrm{dt}, J=17.1,2.1 \mathrm{~Hz}, 1 \mathrm{H}), 3.30(\mathrm{ddd}, J=17.1,7.8,2.1 \mathrm{~Hz}, 1 \mathrm{H}), 3.88(\mathrm{dt}$, $J=7.8,1.8 \mathrm{~Hz}, 1 \mathrm{H}), 4.17-4.25(\mathrm{~m}, 2 \mathrm{H}), 6.24(\mathrm{t}, J=1.8 \mathrm{~Hz}, 1 \mathrm{H}), 7.18(\mathrm{~d}, J=8.1 \mathrm{~Hz}, 2 \mathrm{H}), 7.25-7.40(\mathrm{~m}$, 5H), $7.56(\mathrm{~d}, J=8.1 \mathrm{~Hz}, 2 \mathrm{H}) ;{ }^{13} \mathrm{C}-\mathrm{NMR}\left(\mathrm{CDCl}_{3}\right) \delta 14.4,14.5,21.0,40.2,52.8,59.3,102.4,106.4,124.6$, 125.6, 126.4, 128.4, 128.5, 129.1, 134.8, 137.3, 140.2, 146.3, 166.0, 166.6; IR (neat) 2977, 2923, $1693 \mathrm{~cm}^{-1}$; EI-MS m/z (rel intensity) $360\left([\mathrm{M}]^{+}, 11\right), 314$ (100), 243 (27), 228 (20); HRMS [M] ${ }^{+}$calcd. for $\mathrm{C}_{24} \mathrm{H}_{24} \mathrm{O}_{3}$ : 360.1725 , found 360.1721 .

Ethyl 6a-(4-methoxyphenyl)-2-methyl-5-phenyl-4,6a-dihydro-3aH-cyclopenta[b]furan-3-carboxylate (4e). Yield $154 \mathrm{mg}(82 \%)$. A red oil; TLC (EtOAc/hexanes (1:10)) $R_{\mathrm{f}}=0.50 ;{ }^{1} \mathrm{H}-\mathrm{NMR}\left(\mathrm{CDCl}_{3}\right) \delta 1.29(\mathrm{t}, J=7.2 \mathrm{~Hz}$, 3H), $2.29(\mathrm{~s}, 3 \mathrm{H}), 2.99(\mathrm{dt}, J=17.1,1.8 \mathrm{~Hz}, 1 \mathrm{H}), 3.26(\mathrm{ddd}, J=17.1,6.6,2.1 \mathrm{~Hz}, 1 \mathrm{H}), 3.79(\mathrm{~s}, 3 \mathrm{H}), 3.83$ $(\mathrm{dt}, J=6.6,1.8 \mathrm{~Hz}, 1 \mathrm{H}), 4.13-4.24(\mathrm{~m}, 2 \mathrm{H}), 6.22(\mathrm{t}, J=1.8 \mathrm{~Hz}, 1 \mathrm{H}), 6.88(\mathrm{~d}, J=6.6 \mathrm{~Hz}, 2 \mathrm{H}), 7.25-7.38$ $(\mathrm{m}, 5 \mathrm{H}), 7.53(\mathrm{~d}, J=7.8 \mathrm{~Hz}, 2 \mathrm{H}) ;{ }^{13} \mathrm{C}-\mathrm{NMR}\left(\mathrm{CDCl}_{3}\right) \delta 14.4,14.5,40.1,52.7,55.2,59.4,102.2,106.4,113.7$, $125.5,125.9,126.4,128.4,128.5,134.8,135.3,146.3,159.0,166.0,166.6$; IR (neat) 2931, 1696, $1644 \mathrm{~cm}^{-1}$; EI-MS m/z (rel intensity) $376\left([\mathrm{M}]^{+}, 18\right), 330$ (100), 287 (19), 259 (33); HRMS [M] ${ }^{+}$calcd. for $\mathrm{C}_{24} \mathrm{H}_{24} \mathrm{O}_{4}$ : 376.1675 , found 376.1672 . 
Ethyl 6a-(3-methoxyphenyl)-2-methyl-5-phenyl-4,6a-dihydro-3aH-cyclopenta[b]furan-3-carboxylate (4f). Yield $152 \mathrm{mg}(81 \%)$. A yellow oil; TLC (EtOAc/hexanes $(1: 10)) R_{\mathrm{f}}=0.44 ;{ }^{1} \mathrm{H}-\mathrm{NMR}\left(\mathrm{CDCl}_{3}\right) \delta 1.30(\mathrm{t}$, $J=7.2 \mathrm{~Hz}, 3 \mathrm{H}), 2.32(\mathrm{~s}, 3 \mathrm{H}), 3.02(\mathrm{dt}, J=17.1,2.1 \mathrm{~Hz}, 1 \mathrm{H}), 3.30(\mathrm{ddd}, J=17.1,7.8,2.1 \mathrm{~Hz}, 1 \mathrm{H}), 3.80(\mathrm{~s}$, $3 \mathrm{H}), 3.88(\mathrm{dt}, J=7.8,1.8 \mathrm{~Hz}, 1 \mathrm{H}), 4.16-4.24(\mathrm{~m}, 2 \mathrm{H}), 6.22(\mathrm{t}, J=1.8 \mathrm{~Hz}, 1 \mathrm{H}), 6.80-6.84(\mathrm{~m}, 1 \mathrm{H}), 6.92-6.95$ $(\mathrm{m}, 2 \mathrm{H}), 7.25-7.39(\mathrm{~m}, 4 \mathrm{H}), 7.55(\mathrm{~d}, J=8.1 \mathrm{~Hz}, 2 \mathrm{H}) ;{ }^{13} \mathrm{C}-\mathrm{NMR}\left(\mathrm{CDCl}_{3}\right) \delta 14.4,40.2,52.8,55.2,59.4,102.2$, $106.5,110.7,112.4,116.9,125.4,126.4,128.4,128.5,129.5,134.7,144.8,146.5,159.7,165.9,166.5 ;$ IR (neat) 3056, 2979, $1693 \mathrm{~cm}^{-1}$; EI-MS m/z (rel intensity) $376\left([\mathrm{M}]^{+}, 18\right), 330$ (100), 287 (48), 259 (41); HRMS $[\mathrm{M}]^{+}$calcd. for $\mathrm{C}_{24} \mathrm{H}_{24} \mathrm{O}_{4}: 376.1675$, found 376.1680 .

Ethyl 6a-([1,1'-biphenyl]-3-yl)-2-methyl-5-phenyl-4,6a-dihydro-3aH-cyclopenta[b]furan-3-carboxylate (4g). Yield $180 \mathrm{mg}(85 \%)$. A red oil; TLC (EtOAc/hexanes (1:10)) $R_{\mathrm{f}}=0.53 ;{ }^{1} \mathrm{H}-\mathrm{NMR}\left(\mathrm{CDCl}_{3}\right) \delta 1.33(\mathrm{t}$, $J=7.2 \mathrm{~Hz}, 3 \mathrm{H}), 2.38(\mathrm{~s}, 3 \mathrm{H}), 3.10(\mathrm{dt}, J=17.1,1.8 \mathrm{~Hz}, 1 \mathrm{H}), 3.38(\mathrm{ddd}, J=17.1,6.0,2.1 \mathrm{~Hz}, 1 \mathrm{H}), 3.99(\mathrm{dt}$, $J=6.0,1.8 \mathrm{~Hz}, 1 \mathrm{H}), 4.20-4.28(\mathrm{~m}, 2 \mathrm{H}), 6.32(\mathrm{t}, J=1.8 \mathrm{~Hz}, 1 \mathrm{H}), 7.34-7.49(\mathrm{~m}, 8 \mathrm{H}), 7.53-7.63(\mathrm{~m}, 6 \mathrm{H})$; ${ }^{13} \mathrm{C}-\mathrm{NMR}\left(\mathrm{CDCl}_{3}\right) \delta 14.4,14.5,40.3,52.9,59.4,102.4,106.5,123.4,123.6,125.5,126.4,126.5,127.2,127.3$, 128.4, 128.5, 128.7, 128.9, 134.7, 141.0, 141.5, 143.7, 146.6, 165.9, 166.6; IR (neat) 3060, 2981, $1706 \mathrm{~cm}^{-1}$; EI-MS m/z (rel intensity) $422\left([\mathrm{M}]^{+}, 13\right), 376$ (100), 333 (23), 305 (27); HRMS [M] ${ }^{+}$calcd. for $\mathrm{C}_{29} \mathrm{H}_{26} \mathrm{O}_{3}$ : 422.1882, found 422.1884 .

Ethyl 2-methyl-6a-(naphthalen-2-yl)-5-phenyl-4,6a-dihydro-3aH-cyclopenta[b]furan-3-carboxylate (4h). Yield $167 \mathrm{mg}(84 \%)$. A yellow oil; TLC (EtOAc/hexanes (1:10)) $R_{\mathrm{f}}=0.38 ;{ }^{1} \mathrm{H}-\mathrm{NMR}\left(\mathrm{MHz}, \mathrm{CDCl}_{3}\right) \delta 1.31(\mathrm{t}$, $J=7.2 \mathrm{~Hz}, 3 \mathrm{H}), 2.38(\mathrm{~s}, 3 \mathrm{H}), 3.09(\mathrm{dt}, J=17.1,1.8 \mathrm{~Hz}, 1 \mathrm{H}), 3.37(\mathrm{ddd}, J=17.1,7.8,1.8 \mathrm{~Hz}, 1 \mathrm{H}), 3.98(\mathrm{~d}$, $J=7.8 \mathrm{~Hz}, 1 \mathrm{H}), 4.14-4.29(\mathrm{~m}, 2 \mathrm{H}), 6.34(\mathrm{t}, J=1.8 \mathrm{~Hz}, 1 \mathrm{H}), 7.33-7.49(\mathrm{~m}, 6 \mathrm{H}), 7.58-7.61(\mathrm{~m}, 2 \mathrm{H}), 7.75-7.85$ $(\mathrm{m}, 4 \mathrm{H}) ;{ }^{13} \mathrm{C}-\mathrm{NMR}\left(\mathrm{CDCl}_{3}\right) \delta 14.5,14.6,40.4,52.9,59.5,102.6,106.7,123.2,125.5,126.1,126.3,126.5$, 127.6, 128.2, 128.5, 128.6, 132.8, 133.1, 134.8, 140.4, 146.9, 166.1, 166.8; IR (neat) 3056, 2977, $1698 \mathrm{~cm}^{-1}$; EI-MS m/z (rel intensity) 396 ([M] $\left.]^{+}, 22\right), 350$ (100), 307 (23), 279 (53); HRMS [M] calcd. for $\mathrm{C}_{27} \mathrm{H}_{24} \mathrm{O}_{3}$ : 396.1725, found 396.1733.

\section{Conclusions}

In summary, the synthetic application of hypervalent iodine(III) in the formation of cyclopenta[$[b]$ furans is described. This method provides an alternative route to access the target for those cases where the corresponding diene-aldehyde precursor is not readily available or its use limited by intrinsic potential instability.

Supplementary Materials: Supplementary materials can be accessed at: http:/ /www.mdpi.com/1420-3049/21/ $12 / 1713 /$ s1

Acknowledgments: Financial support from the Ministry of Science and Technology of the Republic of China, Taiwan (MOST 105-2113-M-018-004) is gratefully acknowledged. We also thank the assistance from Chang-Chih Hsieh for the X-ray structure determination.

Author Contributions: M.-H.L. conceived and designed the experiments; Y.-C.C. and S.-H.C. performed the experiments. Y.-F.C. performed the X-ray analysis. T.-H.C. gave insight suggestions.

Conflicts of Interest: The authors declare no conflict of interest.

\section{References and Notes}

1. Winters, M.P.; Teleha, C.A.; Kang, F.-A.; McComsey, D.; O’Neill, J.C.; Hou, C.; Kirchner, T.; Wang, P.; Johnson, D.; Sui, Z. The discovery and SAR of cyclopenta[b]furans as inhibitors of CCR2. Bioorg. Med. Chem. Lett. 2014, 24, 2137-2140. [CrossRef] [PubMed]

2. Teleha, C.A.; Branum, S.; Zhang, Y.; Reuman, M.E.; van der Steen, L.; Verbeek, M.; Fawzy, N.; Leo, G.C.; Winters, M.P.; Kang, F.-A.; et al. Lab-scale preparation of a novel cyclopenta[ $b]$ furan chemokine receptor antagonist. Org. Process Res. Dev. 2014, 18, 1630-1640. [CrossRef]

3. Khan, P.M.; Wu, R.; Bisht, K.S. Pd(0) catalyzed intramolecular alkylation: Stereoselective synthesis of furan and isoxazoline-2-oxide analogs. Tetrahedron 2007, 63, 1116-1126. [CrossRef] 
4. Li, E.; Huang, Y. Phosphine-catalyzed domino reactions: A route to functionalized bicyclic skeletons. Chem. Eur. J. 2014, 20, 3520-3527. [CrossRef] [PubMed]

5. Riveira, M.J.; Mischne, M.P. One-pot organocatalytic tandem Aldol/polycyclization reactions between 1,3-dicarbonyl compounds and $\alpha, \beta, \gamma, \delta$-unsaturated aldehydes for the straightforward assembly of cyclopenta[b]furan-type derivatives: New insight into the Knoevenagel reaction. Chem. Eur. J. 2012, 18, 2382-2388. [CrossRef] [PubMed]

6. Riveira, M.J.; Mischne, M.P. An interrupted vinylogous iso-Nazarov reaction: Cycloisomerization of conjugated trienones to cyclopenta[b]furan derivatives. J. Org. Chem. 2014, 79, 8244-8254. [CrossRef] [PubMed]

7. Wirth, T. (Ed.) Topics in Current Chemistry. Hypervalent Iodine Chemistry; Springer: Berlin, Germany, 2016; Volume 373, pp. 1-309.

8. Zhdankin, V.V. Hypervalent Iodine Chemistry: Preparation, Structure, and Synthetic Applications of Polyvalent Iodine Compounds; Wiley: Chichester, UK, 2014.

9. Wirth, T. Hypervalent iodine chemistry in synthesis: Scope and new directions. Angew. Chem. Int. Ed. Engl. 2005, 44, 3656-3665. [CrossRef] [PubMed]

10. Zheng, Y.; Li, X.; Ren, C.; Zhang-Negrerie, D.; Du, Y.; Zhao, K. Synthesis of oxazoles from enamides via phenyliodine diacetate-mediated intramolecular oxidative cyclization. J. Org. Chem. 2012, 77, 10353-10361. [CrossRef] [PubMed]

11. Lv, J.; Zhang-Negrerie, D.; Deng, J.; Du, Y.; Zhao, K. Metal-free synthesis of 2-oxindoles via $\mathrm{PhI}(\mathrm{OAc})_{2}$-mediated oxidative C-C bond formation. J. Org. Chem. 2014, 79, 1111-1119. [CrossRef] [PubMed]

12. Liu, G.-Q.; Li, Y.-M. Regioselective (diacetoxyiodo)benzene-promoted halocyclization of unfunctionalized olefins. J. Org. Chem. 2014, 79, 10094-10109. [CrossRef] [PubMed]

13. Karade, N.N.; Shirodkar, S.G.; Patil, M.N.; Potrekar, R.A.; Karade, H.N. Diacetoxyiodobenzene-mediated oxidative addition of 1,3-dicarbonyl compounds to olefins: An efficient one-pot synthesis of 2,3-dihydrofuran derivatives. Tetrahedron Lett. 2003, 44, 6729-6731. [CrossRef]

14. Kalpogiannaki, D.; Martini, C.-I.; Nikopoulou, A.; Nyxas, J.A.; Pantazi, V.; Hadjiarapoglou, L.P. Fused dihydrofurans from the one-pot, three-component reaction of 1,3-cyclohexanedione, iodobenzene diacetate and alkenes. Tetrahedron 2013, 69, 1566-1575. [CrossRef]

15. Fan, L.; Chen, W.; Tang, K.; Wu, D. Stereoselective synthesis of $\alpha$-ylidene- $\beta$-dicarbonyl compounds: A mild $\mathrm{PhI}(\mathrm{OAc})_{2}$-mediated dehydrogenation process. Chem. Lett. 2012, 41, 940-942. [CrossRef]

16. Duan, Y.-N.; Cui, L.-Q.; Zuo, L.-H.; Zhang, C. Recyclable hypervalent-iodine-mediated dehydrogenative $\alpha, \beta^{\prime}$-bifunctionalization of $\beta$-keto esters under metal-free conditions. Chem. Eur. J. 2015, 21, 13052-13057. [CrossRef] [PubMed]

17. Lin, M.-H.; Li, Y.-S.; Kuo, C.-K.; Chen, C.-H.; Huang, Y.-C.; Liang, K.-Y.; Chen, Y.-C.; Tsai, C.-H.; Chuang, T.-H. Synthesis of hexahydro- $1 H$-isoindole derivatives from arylacyl bromides via homoallenic bromohydrins. J. Org. Chem. 2015, 80, 2462-2466. [CrossRef] [PubMed]

18. The structure has been deposited with the Cambridge Crystallographic Data Centre (4b: CCDC 1517150). These Data Can be Obtained Free of Charge from The Cambridge Crystallographic Data Centre via. Available online: http:/ / www.ccdc.cam.ac.uk/data_request/cif (accessed on 14 November 2016).

Sample Availability: Samples of the compounds $\mathbf{1 a}-\mathbf{h}, \mathbf{4 a}-\mathbf{h}$ are available from the authors.

(C) 2016 by the authors; licensee MDPI, Basel, Switzerland. This article is an open access article distributed under the terms and conditions of the Creative Commons Attribution (CC-BY) license (http://creativecommons.org/licenses/by/4.0/). 